\title{
Formação inicial do enfermeiro quanto a temática saúde sexual e reprodutiva: revisão integrativa
}

\author{
Initial training of nurses on the theme sexual and reproductive health: integrative review \\ Formación inicial de enfermeras sobre el tema salud sexual y reproductiva: revisión integradora
}

Recebido: 12/11/2021 | Revisado: 24/11/2021 | Aceito: 25/11/2021 | Publicado: 07/12/2021

\author{
Alessandra Leão Brasileiro \\ ORCID: https://orcid.org/0000-0001-5486-2923 \\ Universidade Estadual do Ceará, Brasil \\ E-mail: alessandra.leao.brasileiro@gmail.com \\ Lídia Andrade Lourinho \\ ORCID: https://orcid.org/0000-0002-5883-9007 \\ Universidade Estadual do Ceará, Brasil \\ E-mail: lidiandrade67@gmail.com
}

\begin{abstract}
Resumo
As questões que envolvem saúde sexual e reprodutiva são pouco ou mesmo não são abordadas pelo fato de que os profissionais de saúde referem dificuldades em abordar essa temática e, além do desconforto em falar da sexualidade ou da saúde sexual de seus pacientes, muitos deles não se sentem preparados para exercer esse cuidado. Essa revisão integrativa trata-se de um recorte de uma dissertação. É uma reflexão sistematizada sobre o desenvolvimento de estratégias de articulação entre o mundo do trabalho, da aprendizagem e da sociedade, a partir do reconhecimento de avanços, fragilidades e fortalezas do atual modelo de atenção à saúde tendo como eixo central princípios do SUS e a necessidade de inovação das práticas e da formação em saúde. O objetivo é caracterizar pesquisas que abordem a temática saúde sexual e reprodutiva e que relacione com a formação inicial do enfermeiro. É necessário incentivos a pesquisa e a aplicabilidade sobre saúde sexual e reprodutiva, principalmente com a atualização da nomenclatura e o propósito no que significa o termo garantindo a dignidade e os direitos sexuais e reprodutivos da população. Foi possível concluir que é irrisório a quantidade de artigos que contemplam a termologia saúde sexual e reprodutiva, deixando claro o desfalque nesse assunto que é tão relevante para Saúde Pública. Mesmo assim, as publicações relevantes para a temática são de predominância das pesquisas voltadas para os profissionais da saúde e/ou para a população.
\end{abstract}

Palavras-chave: Educação em enfermagem; Planejamento familiar; Saúde reprodutiva; Saúde sexual.

\begin{abstract}
The issues involving sexual and reproductive health are little or even not addressed by the fact that health professionals report difficulties in addressing this theme and, in addition to the discomfort in talking about sexuality or the sexual health of their patients, many of them do not feel prepared to exercise this care. This integrative review is a clipping of a dissertation. It is a systematized reflection on the development of strategies of articulation between the world of work, learning and society, based on the recognition of advances, weaknesses and strengths of the current model of health care having as its central axis principles of the SUS and the need for innovation of health practices and training. The objective is to characterize research that addresses the theme of sexual and reproductive health and that relates to the initial training of nurses. It is necessary to encourage research and applicability on sexual and reproductive health, especially with the updating of the nomenclature and the purpose in what the term means guaranteeing the dignity and sexual and reproductive rights of the population. It was possible to conclude that it is derisory the amount of articles that contemplate the termology sexual and reproductive health, making clear the embezzlement in this subject that is so relevant to Public Health. Even so, the publications relevant to the theme are predominantly research aimed at health professionals and/or the population.
\end{abstract}

Keywords: Nursing education; Family planning; Reproductive health; Sexual health.

\section{Resumen}

Los temas relacionados con la salud sexual y reproductiva son poco o incluso no abordados por el hecho de que los profesionales de la salud informan dificultades para abordar este tema y, además de la incomodidad al hablar sobre la sexualidad o la salud sexual de sus pacientes, muchos de ellos no se sienten preparados para ejercer este cuidado. Esta revisión integradora es un recorte de una disertación. Es una reflexión sistematizada sobre el desarrollo de estrategias de articulación entre el mundo del trabajo, el aprendizaje y la sociedad, a partir del reconocimiento de los avances, debilidades y fortalezas del modelo actual de atención en salud teniendo como eje central los principios del SUS y la necesidad de innovación de las prácticas y capacitaciones en salud. El objetivo es caracterizar investigaciones que 
aborden el tema de la salud sexual y reproductiva y que se relacionen con la formación inicial de enfermeras. Es necesario fomentar la investigación y aplicabilidad en materia de salud sexual y reproductiva, especialmente con la actualización de la nomenclatura y el propósito en lo que significa el término garantizar la dignidad y los derechos sexuales y reproductivos de la población. Se pudo concluir que es irrisoria la cantidad de artículos que contemplan la termología de la salud sexual y reproductiva, dejando en claro la malversación en este tema que es tan relevante para la Salud Pública. Aun así, las publicaciones relevantes para el tema son predominantemente investigaciones dirigidas a profesionales de la salud y/o a la población.

Palabras clave: Educación en enfermería; Planificación familiar; Salud reproductiva; Salud sexual.

\section{Introdução}

A Organização Mundial de Saúde (OMS) define saúde sexual como um "estado de bem-estar físico, emocional, mental e social em relação à sexualidade" e ainda reitera que, dentre outros aspectos, abraça também a possibilidade de ter uma vida sexual agradável e segura, livre de coerção, discriminação e violência (WHO, 2015).

Estando a saúde reprodutiva e o planejamento familiar dentro deste conceito ampliado de saúde sexual que, segundo Rocha et al. (2016) é uma forma de assegurar que os cidadãos tenham acesso à informação, aos serviços de saúde e aos métodos contraceptivos eficazes e seguros, de maneira a permitir uma sexualidade segura e saudável. Também permite a decisão de ter ou não filhos, e quando tê-los.

No Brasil, o planejamento familiar foi definido por lei como um "conjunto de ações de regulação da fecundidade que visa garantir os direitos iguais de constituição, limitação ou aumento da prole pela mulher, homem ou casal" (Ministério da Saúde, 1996). Porém, passou-se a adotar o termo planejamento reprodutivo, em publicações ministeriais brasileiras, como substituto de planejamento familiar, pois segundo Paiva e Caetano (2020) trata-se de um termo mais abrangente em relação aos direitos sexuais e reprodutivos.

A atenção em saúde sexual e reprodutiva pode ser realizada em grupo ou não e deve abranger a assistência clínica, o aconselhamento e as atividades educativas, estendendo-se ao pré-natal, parto e puerpério, à assistência humanizada ao aborto, ao atendimento às infecções sexualmente transmissíveis (IST's) e outras afecções do aparelho reprodutor, envolvendo todas as populações, incluindo lésbicas, gays, bissexuais, travestis, transexuais, transgêneros ou intersexuais (LGBTI); à promoção da sexualidade humana; ao fortalecimento do exercício da paternidade responsável, bem como o encaminhamento e orientações quanto ao tratamento apropriado, em casos de infertilidade (ONU, 1994; Ministério da Saúde, 2013).

Tratando-se de uma abordagem que abrange o exercício da vivência da sexualidade sem constrangimento, da maternidade voluntária e da anticoncepção auto decidida, essa dimensão surge como um tema polêmico e como grande dificuldade para progresso, devido aos tabus e preconceitos que permeiam a discussão (Lemos, 2014).

É sabido que, no geral, as questões que envolvem saúde sexual e reprodutiva são pouco ou mesmo não são abordadas pelo fato de que os profissionais de saúde referem um certo grau de dificuldade em abordar essa temática e, além do desconforto em falar da sexualidade ou da saúde sexual de seus pacientes, muitos deles não se sentem preparados para exercer esse cuidado (Ministério da Saúde, 2013; Lemos, 2014; Santos et al., 2016; Santos et al., 2019).

Esse despreparo relatado refere-se, muitas vezes, a uma deficiência na abordagem dessa temática logo na graduação que tende a construir sua estrutura curricular de modo que esse assunto seja, muitas vezes, negligenciado (Telo \& Witt, 2018). Sendo, portanto, uma bola de neve que reflete uma distância considerável entre o que é preconizado nas ações de atenção ao planejamento reprodutivo e o que é, de fato, vivenciado nos serviços de saúde.

Destaca-se que a formação de profissionais no Brasil sofre alguns percalços, pois o país possui enormes carências educacionais, o que é agravado ainda mais se considerar sua extensa área geográfica (Bezerra et al., 2019). Em contrapartida, o mercado de trabalho faz constantes exigências em relação à competência dos profissionais atuantes. 
Deste modo, solicita-se que haja uma preocupação em oferecer uma educação superior qualificada para o desenvolvimento de conhecimentos e competências no tempo e com os métodos adequados (Matia et al, 2018).

Neste contexto, o uso de tecnologias educacionais validadas torna-se importante, pois podem ser utilizadas para transformar a metodologia dos professores em suas respectivas áreas de conhecimento e tornar a sala de aula mais convidativa e instigante, aumentando a motivação do discente para aprender (Oliveira et al., 2018).

O termo tecnologia educacional é definido por Litwin (1997) como um corpo de conhecimentos baseado nas disciplinas científicas às práticas de ensino. Esses instrumentos podem ser materializados em manuais, folders, cartilhas, álbuns seriados e têm sido cada vez mais importantes na atenção em saúde, sendo construídos e validados para utilização em diversos contextos.

Percebe-se que, particularmente, as cartilhas educacionais são importantes ferramentas no fornecimento e construção de conhecimento e amplamente utilizados como material orientador, uma vez que aparecem em diversos meios de comunicação, em especial no meio educacional como recurso didático-pedagógico por profissionais da área de saúde e professores em sala de aula (Ramos \& Araújo, 2017).

Essa pesquisa trata-se de um recorte de uma dissertação e tem como justificativa científica a necessidade de produções e abordagens didáticas voltadas à formação e preparos dos profissionais de enfermagem para o trabalho com o assunto saúde sexual e reprodutiva.

Apontar-se como os objetos de estudo desta pesquisa: saúde sexual e reprodutiva e formação em saúde. A questão de pesquisa foi estabelecida de acordo com a estratégia PCC, sendo "P" - população: discentes do curso de graduação em enfermagem; "C" - conceito: formação inicial; "C" - contexto: saúde sexual e reprodutiva. Tendo como questão: "Como a temática saúde sexual e reprodutiva perpassa a formação inicial do enfermeiro?”

Acredita-se na relevância dessa pesquisa por se tratar de uma reflexão sistematizada sobre o desenvolvimento de estratégias de articulação entre o mundo do trabalho, da aprendizagem e da sociedade, a partir do reconhecimento de avanços, fragilidades e fortalezas do atual modelo de atenção à saúde tendo como eixo central princípios do SUS e a necessidade de inovação das práticas e da formação em saúde.

O objetivo dessa pesquisa é caracterizar estudos que abordem a temática saúde sexual e reprodutiva e que relacione a discentes da graduação de enfermagem, identificando como a temática saúde sexual e reprodutiva perpassa a formação inicial do enfermeiro.

\section{Metodologia}

A revisão integrativa é uma metodologia que permite a sintetização de uma variedade de pesquisas com resultados de aplicação relevante para a prática e para a teorização, além de ser um instrumento válido da prática baseada em evidências, principalmente no cenário atual da enfermagem no Brasil (Souza, et al., 2010).

É denominada integrativa porque fornece informações mais amplas sobre um tema, constituindo, assim, um corpo de conhecimento. Deste modo, o autor pode elaborar uma revisão integrativa com diferentes finalidades, podendo ser direcionada para a definição de conceitos, revisão de teorias ou análise metodológica dos estudos incluídos de um tópico particular (Mendes, et al., 2008).

Souza, et.al. (2010) ainda afirma que esse método tem seus desafios e complexidade, e é requerido do pesquisador disciplina e organização durante o processo de coleta de dados, com uma inclusão sistemática, principalmente da análise de dados, o que diminuirá a ocorrência de erros.

Para a construção da revisão integrativa é preciso percorrer seis etapas distintas, sendo elas a identificação do tema e seleção da hipótese ou questão de pesquisa; estabelecimento de critérios para inclusão e exclusão de estudos/amostragem ou 
busca na literatura; definição das informações a serem extraídas dos estudos selecionados/ categorização dos estudos; avaliação dos estudos incluídos; interpretação dos resultados; e apresentação da revisão/síntese do conhecimento (Mendes, et. al., 2008).

Segundo o autor Souza, et. al. (2010) o levantamento bibliográfico é uma das principais formas de iniciar uma pesquisa, traçando semelhanças e diferenças entre os artigos encontrados. A revisão de literatura de pesquisa tem como propósito geral reunir conhecimentos sobre um tópico, ajudando nas fundações de um estudo significativo para enfermagem.

Para a construção do conhecimento acerca da temática a formação do discente de enfermagem e saúde sexual e reprodutiva foram utilizadas as bases de dados Scientific Electronic Library Online (SCIELO), sendo a plataforma de dados com destaque por apresentar as literatura relevantes para a pesquisa, Literatura Latino-Americana e do Caribe em Ciências da Saúde (LILACS) e Medical Literature Analysis and Retrieval Sistem on-line (Medline), plataforma de busca com o maior número de artigos, como será apresentado posteriormente. Além de fundamentos como relatórios governamentais, biblioteca virtual do Ministério da Saúde, como fontes de pesquisa para o levantamento das literaturas pertinentes ao estudo.

Os descritores utilizados foram retirados do Banco de Descritores em Ciências da Saúde - DECS, sendo eles: saúde sexual e saúde reprodutiva, saúde sexual e reprodutiva, planejamento familiar, educação em enfermagem. E no Banco de descritores do Medical Subject Headings - MeSH: direitos sexuais e reprodutivos, serviços de saúde reprodutiva, saúde reprodutiva, comportamento reprodutivo, comportamento sexual. E foram empregados os operadores booleanos "OR" e “AND” e o recurso de truncamento “*” na operacionalização da coleta da amostra. No Quadro 1 é apresentado a estratégias de busca utilizadas nas respectivas bases de dados.

Quadro 1 - Estratégias de busca utilizadas nas respectivas bases de dados.

\begin{tabular}{|l|l|}
\hline BASE DE DADOS & \multicolumn{1}{c|}{ SINTAXE DE PESQUISA } \\
\hline SCIELO & $\begin{array}{l}\text { (saúde sexual) AND (saúde reprodutiva) AND (enfermagem); } \\
\text { planejamento familiar; } \\
\text { educação em enfermagem saúde sexual; } \\
\text { direito sexual e reprodutivo. }\end{array}$ \\
\hline LILACS & $\begin{array}{l}\text { saúde AND sexual AND saúde AND reprodutiva AND enfermagem; } \\
\text { planejamento familiar; } \\
\text { educação em enfermagem saúde sexual; } \\
\text { direito sexual e reprodutivo. }\end{array}$ \\
\hline MEDLINE & $\begin{array}{l}\text { saúde AND sexual AND saúde AND reprodutiva AND enfermagem; } \\
\text { planejamento AND familiar; } \\
\text { educação AND em AND enfermagem AND saúde AND sexual; } \\
\text { direito AND sexual AND e reprodutivo. }\end{array}$ \\
\hline
\end{tabular}

Fonte: Alessandra Leão Brasileiro (2021).

\section{Resultados}

A busca deu-se do período de julho a setembro do ano de 2021. No total foram encontrados em média 48.600 materiais científicos que perpassavam pela temática saúde sexual e reprodutiva. Sendo 133 artigos da base de dados LILACS, 48.147 publicações foram encontradas na MEDLINE e 320 pesquisas estavam na plataforma da SCIELO. As informações foram resumidas na Figura 1 que consta o fluxograma feito a partir do método PRISMA (Moher, Liberati, Tetzlaff, Altman, \& The PRISMA Group, 2009). 
Figura 1 - Fluxograma do processo de seleção dos artigos acerca da saúde sexual e saúde reprodutiva quanto ao processo de ensino em saúde com profissionais enfermeiro ou discentes do curso de graduação em enfermagem.
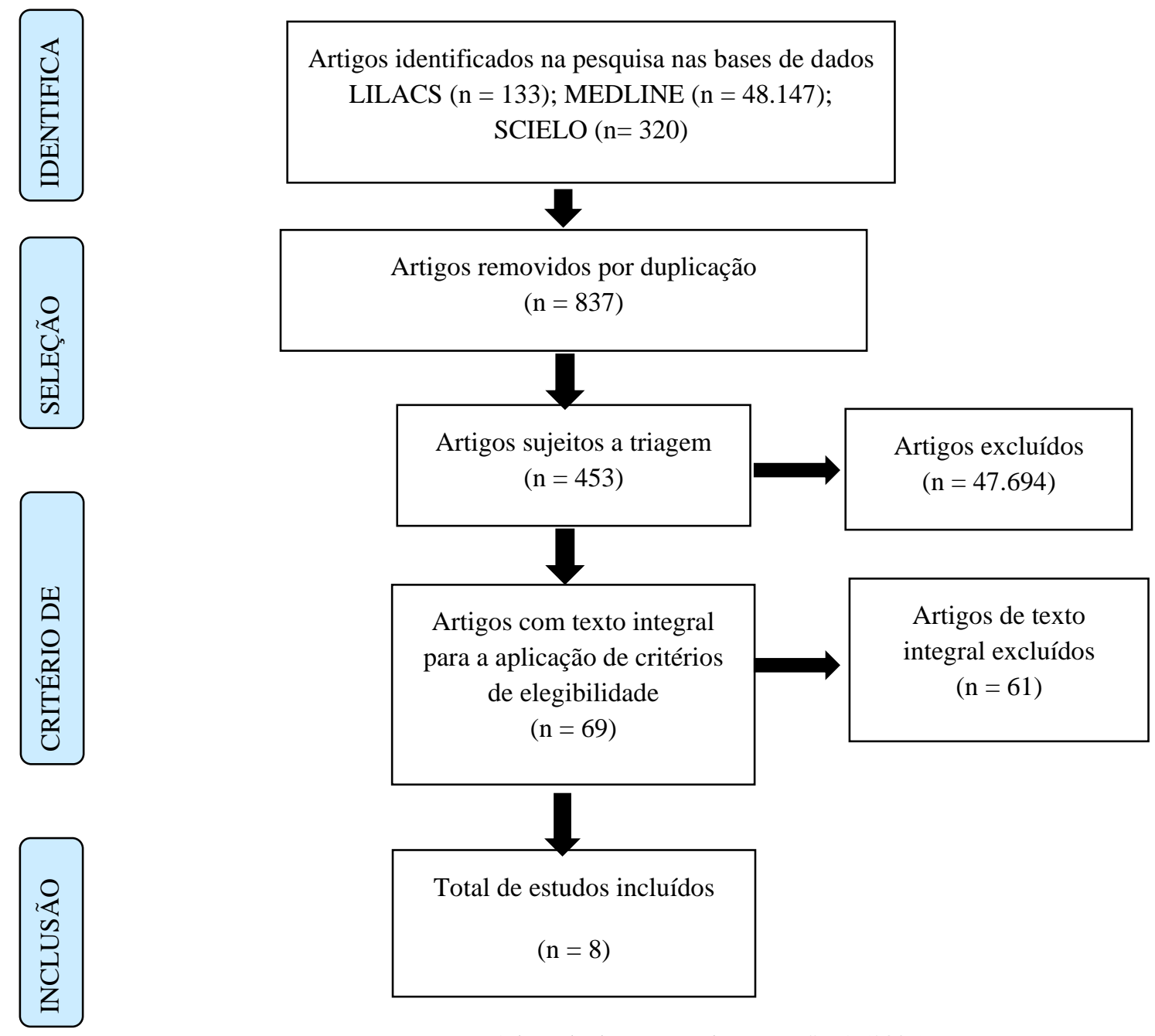

Fonte: Adaptado da recomendação PRISMA (2009).

Os critérios de inclusão adotados para o desenvolvimento da pesquisa foram publicações com a temática saúde sexual e reprodutiva, que também trabalhassem o processo de ensino em saúde com profissionais enfermeiro ou discentes do curso de graduação em enfermagem, disponíveis na íntegra gratuitamente, com o texto completo, publicações nacionais, disponíveis na língua portuguesa, independente do ano de publicação, não foi limitado um recorte temporal, com intuito de ampliar as possibilidades de resultados.

Foram excluídos aqueles que não atendiam aos critérios de inclusão, publicações duplicadas, anais de eventos científicos (resumos), bem como estudos que não abordassem a temática relevante para o objetivo da pesquisa.

Do total de 48.600 artigos encontrados nas três plataformas, foram excluídas 837 publicações por estarem duplicadas, 47.694 publicações foram excluídas por não serem relevante para a pesquisa. Dos 69 artigos considerados elegíveis, foram selecionadas 8 publicações por atenderam aos critérios de inclusão e ao propósito da temática trabalhada na pesquisa.

A seguir é traçado uma relação de base de dados e total de artigos encontrado para os descritores trabalhados na pesquisa, Saúde sexual e reprodutiva, planejamento familiar, educação em enfermagem saúde sexual, direito sexual e reprodutivo.

Como representado no Gráfico 1, para o descritor Saúde Sexual e Reprodutiva, forma encontrados nas três bases de 
dados, LILACS, SCIELO e MEDLINE, um total de 222 artigos, onde 41 publicações (18\%) foram achadas na base de dados SCIELO; 33 artigos (15\%) na fonte LILACS e 148 (67\%) pesquisar na MEDLINE.

Gráfico 1 - Descritores - Saúde Sexual e reprodutiva.

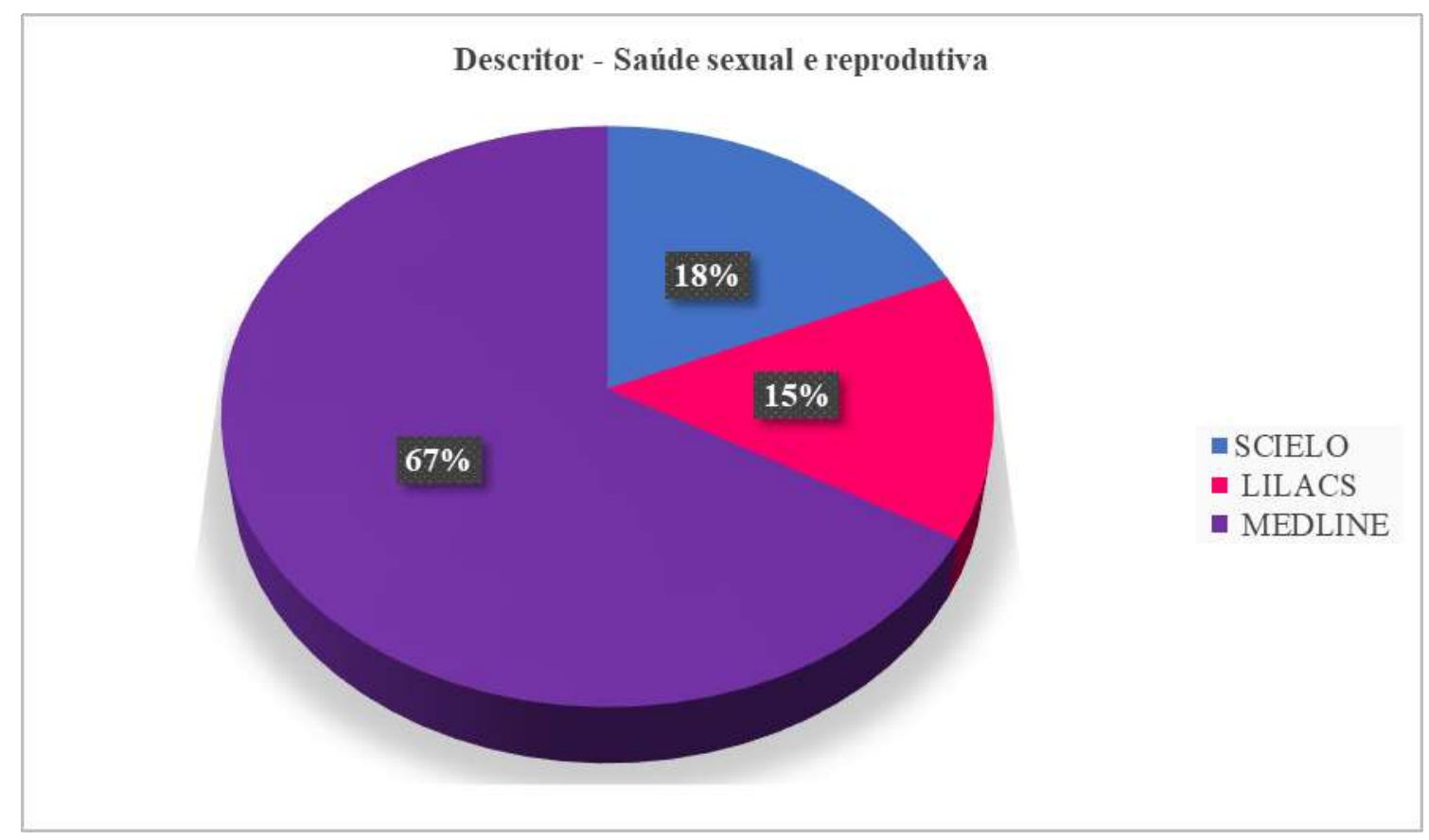

Fonte: Alessandra Leão Brasileiro (2021).

Já com o descritor Planejamento Familiar, foram encontrados nas três plataformas o total de 46.564 artigos, onde 47 publicações eram da LILACS e 220 artigos eram da base SCIELO, ambos, na amostra, conforme o gráfico, correspondem a 0\% dos artigos em relação aos levantados na base MEDLINE, que corresponderam a 99\% dos artigos com a temática planejamento familiar, totalizando em 46.297 artigos. Representação no Gráfico 2. 
Gráfico 2 - Descritor - planejamento Familiar.

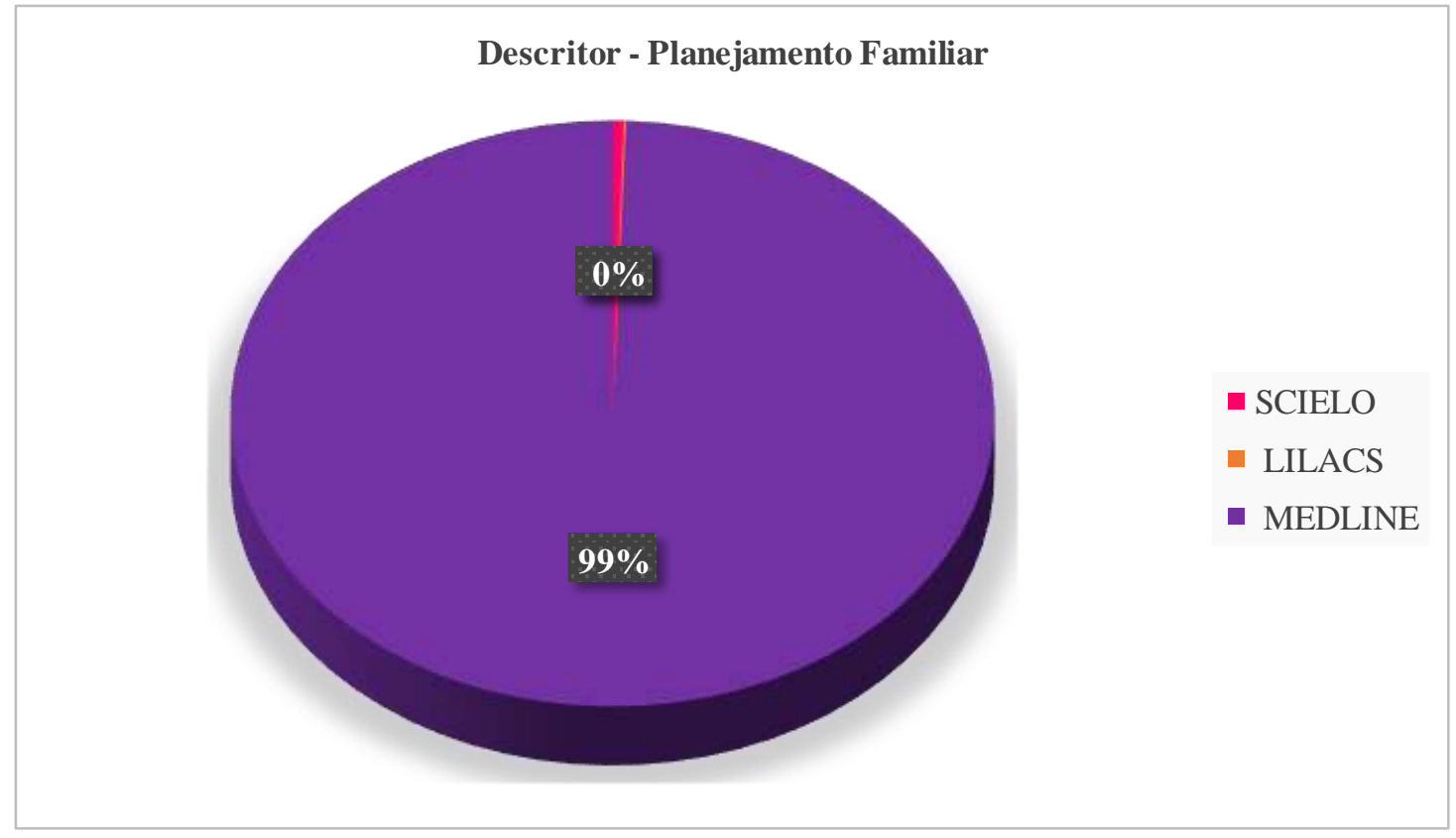

Fonte: Alessandra Leão Brasileiro (2021).

A busca com o descritor Educação em Enfermagem Saúde Sexual teve uma amostra total de 1.111 artigos, como representado no gráfico abaixo, Gráfico 3, SCIELO com 55 publicações e LILACS, 51 artigos, apresentaram 5\%, cada, dos artigos com o descritor supracitado. E novamente a plataforma MEDLINE apresenta um maior número de publicações disponíveis com o descritor em questão, sendo o total de 1.005 artigos que corresponde a $90 \%$.

Gráfico 3 - Descritor - Educação em Enfermagem Saúde Sexual.

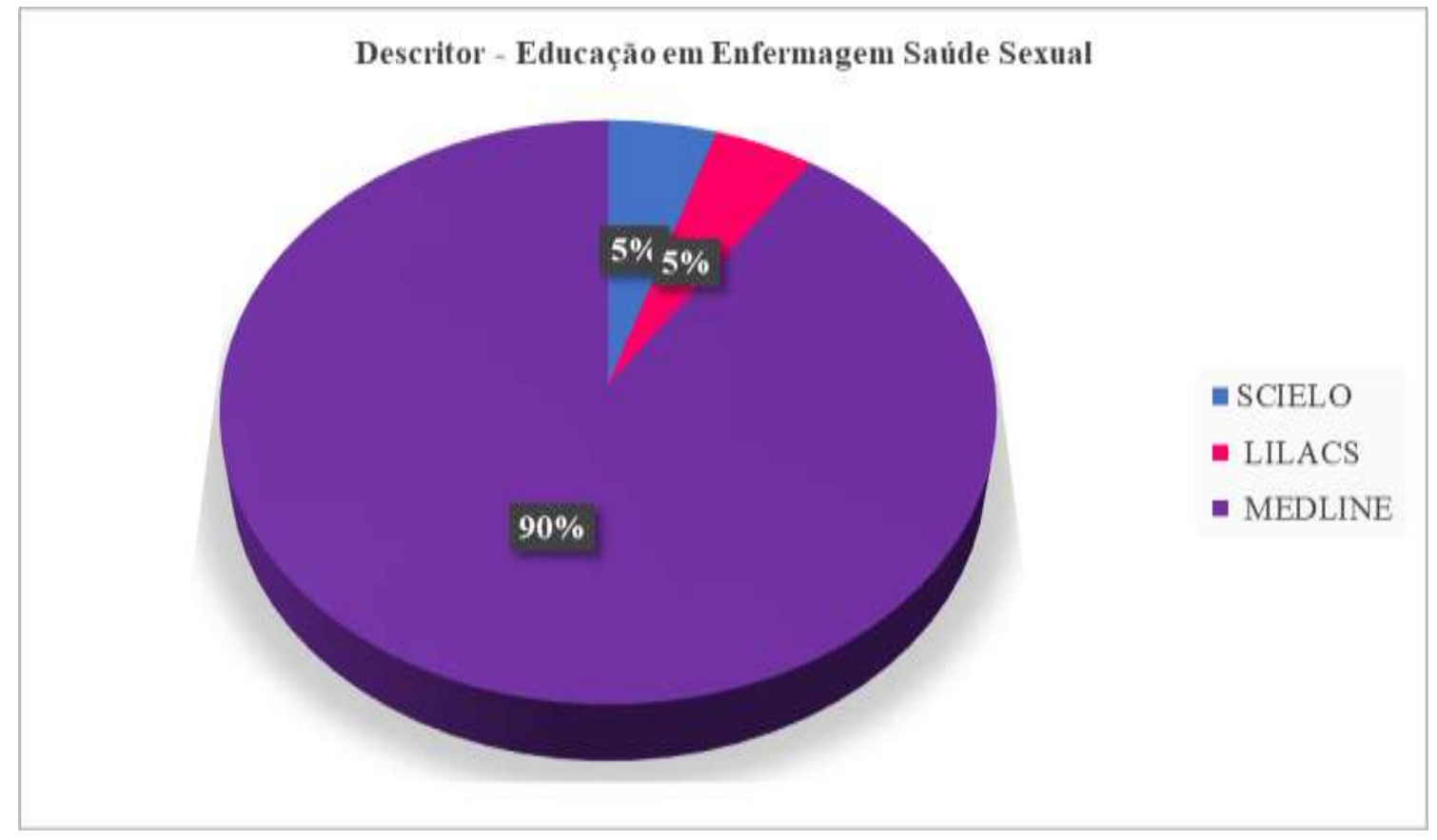

Fonte: Alessandra Leão Brasileiro (2021). 
E por fim, o descritor direito sexual e reprodutivo, teve uma amostra total das três bases de 703 publicações, onde 4 artigos (0\%) estavam disponíveis na plataforma SCIELO, LILACS continha 2 artigos (0\%) e MEDLINE com 697 pesquisas (99\%), como representado no Gráfico 4.

Gráfico 4 - Descritor - Direito Sexual e Reprodutivo.

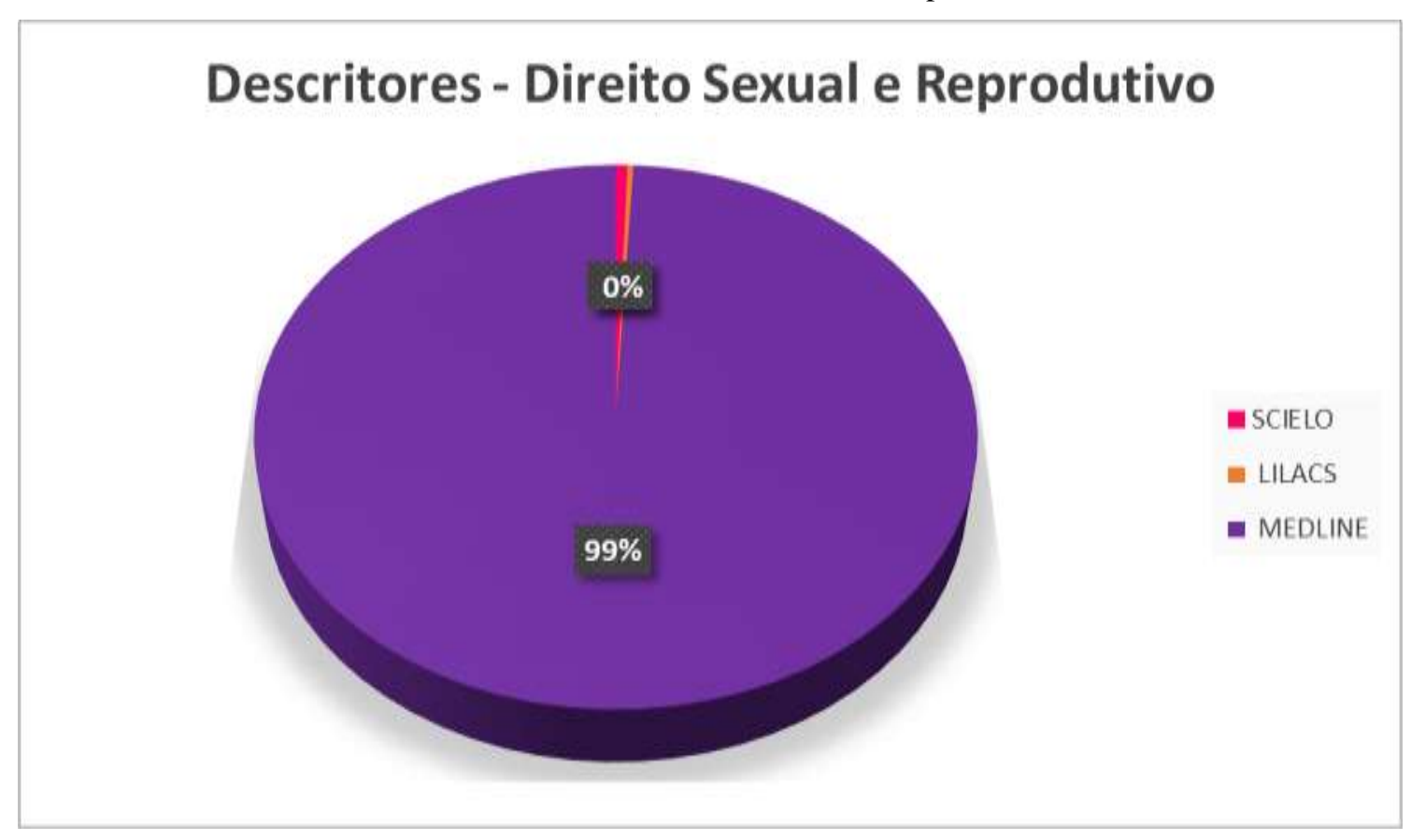

Fonte: Alessandra Leão Brasileiro (2021).

Os artigos da amostra foram selecionados por meio da sequência: leitura de título-resumo e leitura do texto integral. E as literaturas foram elencadas e organizadas no Quadro 2, onde são levantadas informações pertinentes das publicações. 
Quadro 2 - Literaturas fontes para a pesquisa da temática saúde sexual e reprodutiva.

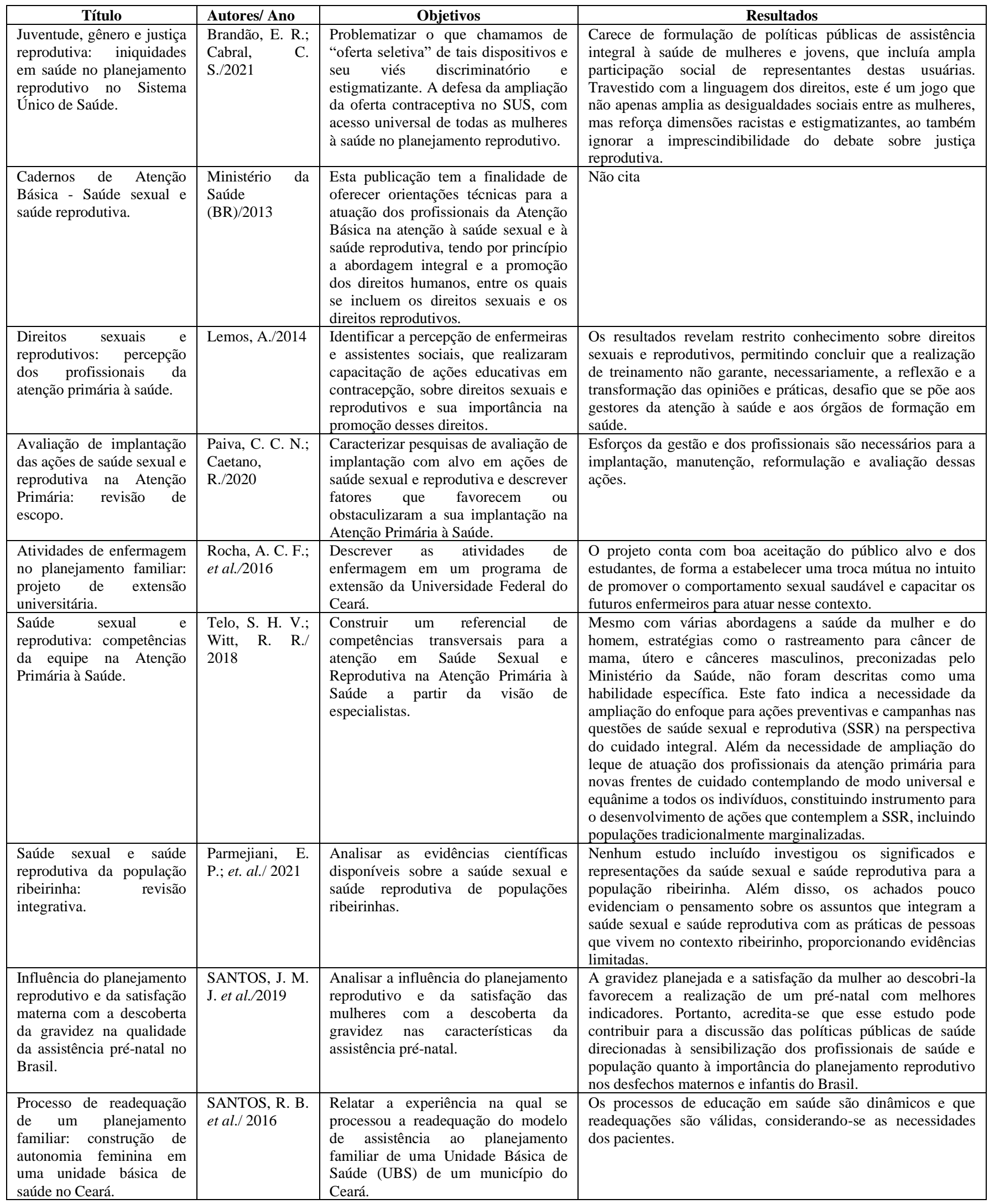

Fonte: Alessandra Leão Brasileiro (2021).

Após a aplicação dos critérios de inclusão e exclusão, foram utilizados um total de 09 referencias, variando do ano de 2013 ao ano de 2021, representação no Gráfico 5. Sendo 08 artigos científicos, tendo como autores Brandão e Cabral (2021); 
Lemos (2014); Paiva e Caetano (2020); Rocha et.al. (2016); Telo e Witt (2018); Parmejiani, et.al. (2021); Santos, et al. (2016); Santos, et al. (2019) e uma publicação do Ministério da Saúde tratando-se de um caderno de atenção básica sobre saúde sexual e saúde reprodutiva do ano de 2013.

Gráfico 5 - Variação do ano de publicação dos artigos elencados para a pesquisa.

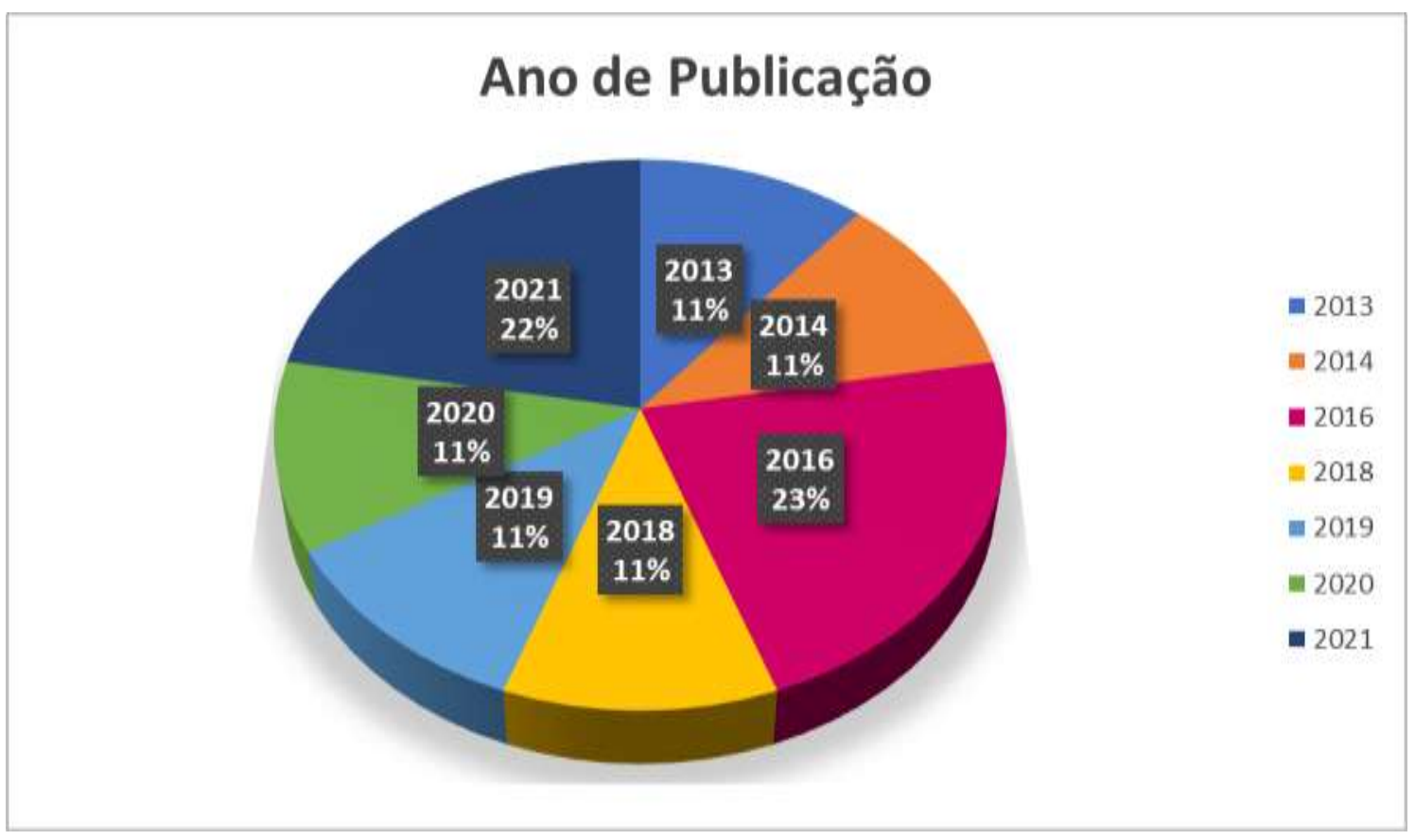

Fonte: Alessandra Leão Brasileiro (2021).

\section{Discussão}

Durante a busca realiza a luz da literatura foi pouco encontrado artigos voltados para o preparo dos discentes para lidar com a temática saúde sexual e reprodutiva, em sua grande maioria são pesquisas voltadas para a população e/ou os profissionais enfermeiros e demais integrantes da equipe multiprofissional, como explanado no Gráfico 6 - Processo de ensino sobre saúde sexual e reprodutiva.

Gráfico 6 - Processo de ensino sobre saúde sexual e reprodutiva.

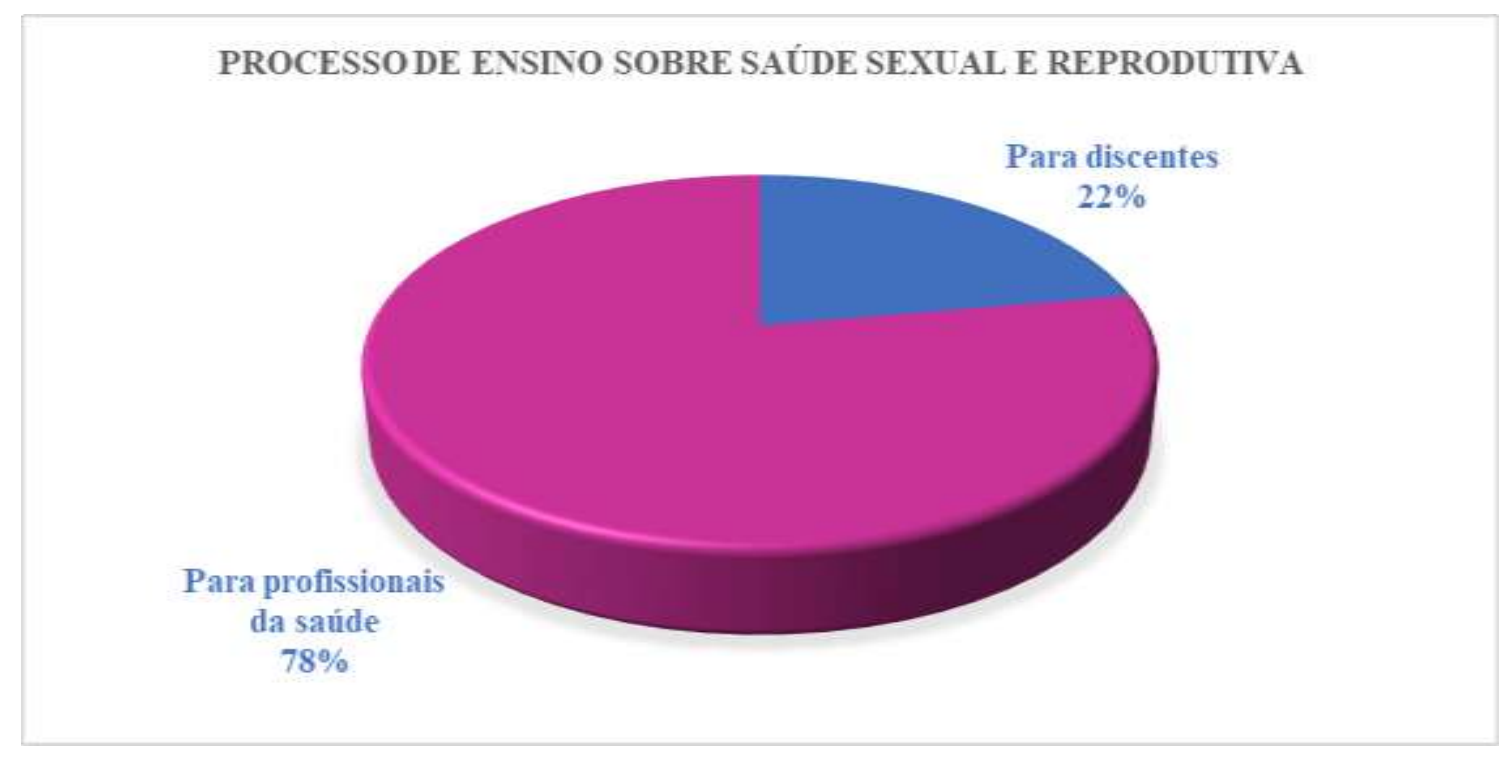

Fonte: Alessandra Leão Brasileiro (2021). 
Dos artigos selecionados como relevantes para o estudo, $78 \%$, ou seja, 7 referencias, trata-se de pesquisas voltadas para os profissionais da saúde e apenas $22 \%$, que correspondem a 2 artigos, abordam a temática saúde sexual e reprodutiva voltada para ensino em saúde de discentes.

Aqueles que atendem a perspectiva do contato teórico-prático do discente com o assunto em questão como o estudo desenvolvido por Rocha et. al. (2016); Santos et. al. (2016), traze excelentes resultados quanto ao aproveitamento e ganho de conhecimento dos discentes, além da satisfação dos usuários quanto ao atendimento. Contudo, é pertinente ressaltar que há necessidade do preparo dos acadêmicos quanto ao conteúdo teórico para que se tenha um melhor rendimento em campo prático, e a longo prazo na vivência profissional.

Por outro ângulo, autores como Paiva e Caetano (2020); Lemos (2013); Santos et. al. (2019); Santos et. al. (2016) mostram a ineficiência estrutural das instituições de atendimento à saúde, do gerenciamento e falta de preparo dos profissionais enfermeiros em compreender a temática saúde sexual e reprodutiva e lidar com as diversas possibilidades que o assunto amplia para o atendimento com os usuários.

Os estudos também nos permitem a identificação da falta de conhecimento por parte da população acerca dos objetos do atendimento à saúde sexual e reprodutiva, como constatado por Brandão e Cabral (2021). Isso também é um reflexo direto da ineficiência do atendimento dos enfermeiros no que se entende por educação em saúde. Podendo ser justificada pelo restrito conhecimento desses profissionais sobre os direitos sexuais e reprodutivos da população como constatado por Lemos (2014).

Durante a busca foram utilizadas as termologias planejamento familiar e saúde sexual e reprodutiva. Mesmo este termo sendo o mais atual, aquele ainda é bastante aplicado, como demostrado no Gráfico 7. O que pode ser algo contestável, já que o termo planejamento familiar restringe bastante o real propósito no que se entende direito sexual e reprodutivo.

Gráfico 7 - Relação de predominância do termo planejamento familiar em relação a nomenclatura saúde sexual e reprodutiva.

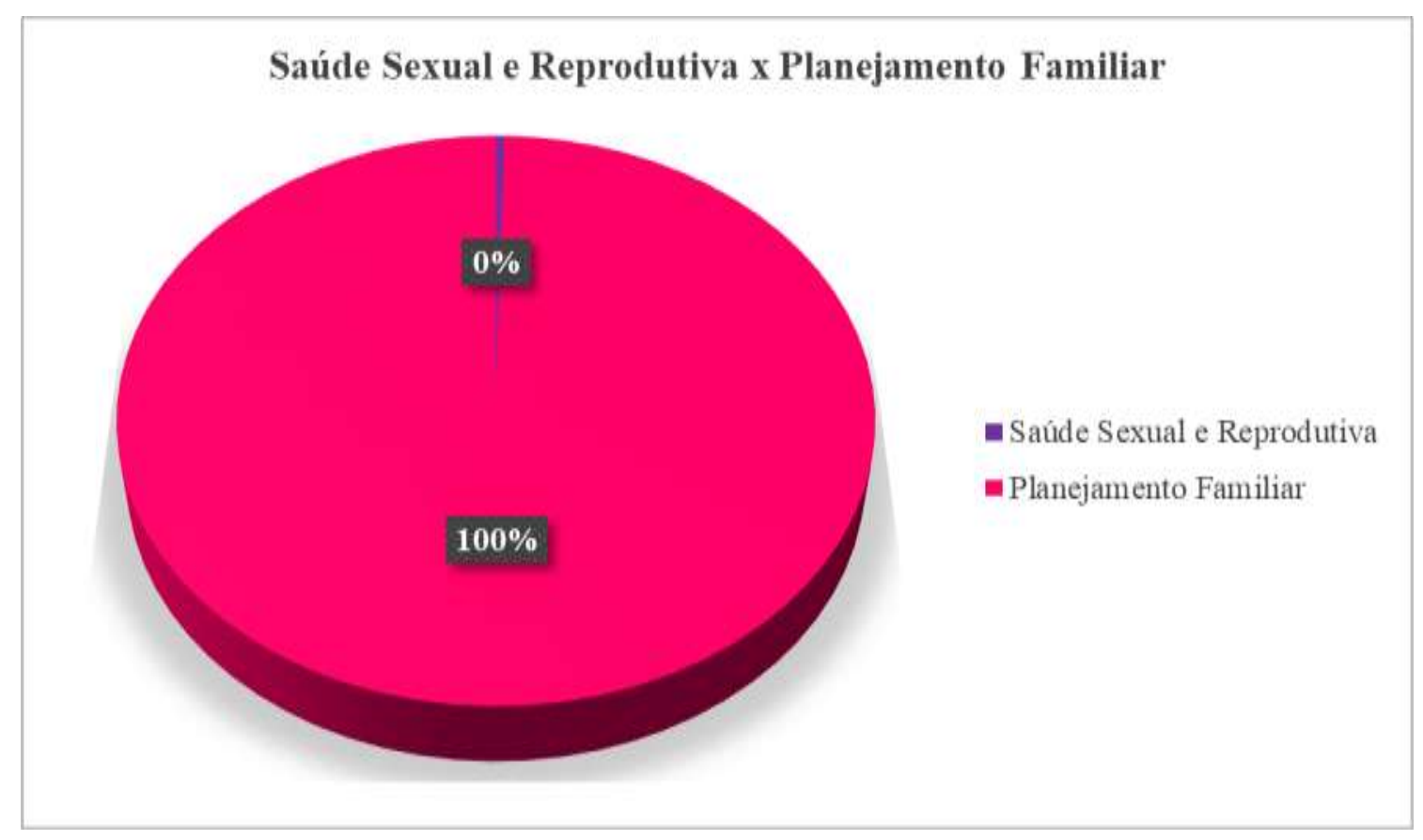

Fonte: Alessandra Leão Brasileiro (2021).

É visível que ainda há grandes fragilidades no preparo dos profissionais enfermeiros para lidar com a pluralidade da população principalmente no que se entende de saúde sexual e reprodutiva. E que também é um fato a carência de estudos e pesquisas sobre a temática, como citado pelos autores Telo e Witt (2018); Parmejiani et al. (2021), que mostram o quanto os 
assuntos diversidade, direitos e segurança sexual e reprodutiva ainda são estigmatizados tanto pela população quanto pelos próprios profissionais.

E se faz necessário incentivos a pesquisa e a aplicabilidade sobre saúde sexual e reprodutiva, principalmente com a atualização da nomenclatura e o propósito no que significa o termo garantindo a dignidade e os direitos sexuais e reprodutivos da população.

Outra observação feita e já citada em parágrafos anteriores, é a escassez de trabalhos voltados para a construção de estratégias facilitadoras do processo de ensino e aprendizagem dos discentes de enfermagem e o preparo dos docentes e acadêmicos para lidar com a temática saúde sexual e reprodutiva e os diversos desafios, peculiaridades e questões que o assunto possibilita quando trabalhado, tanto na abordagem aluno-facilitador como na relação acadêmico-população.

\section{Considerações Finais}

Há uma demanda satisfatória de artigos científicos que passeiam pela temática planejamento familiar, porém, a grande maioria dessas publicações são com enfoque na população e/ou nos profissionais da saúde.

O termo supracitado, planejamento familiar, desfalca o real significado e propósito do que hoje entende-se por saúde sexual e reprodutiva e direitos sexual e reprodutivo.

É irrisório a quantidade de artigos que contemplam a termologia saúde sexual e reprodutiva, deixando claro o desfalque nesse assunto que é tão relevante para Saúde Pública. Mesmo assim, as publicações relevantes para a temática são de predominância das pesquisas voltadas para os profissionais da saúde e/ou para a população, e a maioria dos autores concordam e destacam a falta de preparo e conhecimento por parte dos profissionais acerca do assunto saúde sexual e reprodutiva, o que, consequentemente, reflete na assistência prestada a população.

Dentre vários temas relacionados a saúde, o mote saúde sexual e reprodutiva traz um leque de assuntos que em sua maioria ainda são provocadores de vergonha, medo e desconforto em alguns profissionais da saúde, o que defasa a qualidade da assistência prestada à população.

Segundo a resolução de $n^{\circ} 573$, de 31 de janeiro de 2018, afirma que os cursos de graduação na saúde, conforme as Diretrizes Curriculares Nacionais (DCN), devem promover um ensino-aprendizagem com a abordagem multiprofissional e interdisciplinar, embasado nos conceitos do SUS, focando na integralidade da assistência e atendendo as necessidades em saúde da população.

A resolução ainda ressalta que o enfermeiro, além de gerenciar/administrar, prestar a assistência, também tem um papel fundamental na educação em saúde. Ou seja, a formação do enfermeiro tem que dialogar com o preparo desses profissionais, para que eles consigam educar e orientar da melhor forma possível os clientes, independente do assunto que será abordado.

\section{Referências}

Brandão, E. R., \& Cabral, C. S. (2021). Juventude, gênero e justiça reprodutiva: iniquidades em saúde no planejamento reprodutivo no Sistema Único de Saúde. Artigo Article. Ciência \& Saúde Coletiva, 26(7), 2673-2682. 10.1590/1413-81232021267.08322021.

Bezerra, M. A. A. et al. (2019). Construção e Validação de Tecnologia Educativa para Promoção do Processo Ensino-Aprendizagem. Id On Line Revista de Psicologia, 13(47), 465-477

Lemos, A. (2014). Direitos sexuais e reprodutivos: percepção dos profissionais da atenção primária à saúde. Saúde Debate, 38(101), $244-253$.

Litwin, E. (1997). Tecnologia educacional: política, história e propostas. Artes Médicas, 1997.

Mattia, B. J., Kleba, M. E., \& Prado, M. L. Formação em enfermagem e a prática profissional: uma revisão integrativa da literatura. Rev. Bras. Enferm., 71(4), 2039-2049. 
Mendes, K. D. S., Silveira, R. C. C. P., \& Galvão, C. M. (2008) Revisão integrativa: método de pesquisa para a incorporação de evidências na saúde e na enfermagem. Texto Contexto Enferm. 17(4):758-64.

Ministério da Saúde (2018). Conselho Nacional de Saúde. Resolução n ${ }^{\circ}$ 573/18. Resolução 573 de 31 de janeiro de 2018 . Recomendações do Conselho Nacional de Saúde (CNS) à proposta de Diretrizes Curriculares Nacionais (DCN) para o curso de graduação Bacharelado em Enfermagem. Brasil.

Ministério da Saúde (1996). Lei no 9.263, de 12 de janeiro de 1996. Regula o $§ 7^{\circ}$ do art. 226 da Constituição Federal, que trata do planejamento familiar, estabelece penalidades e dá outras providências. Brasília, DF: Diário Oficial da União.

Ministério da Saúde (2013). Ministério da Saúde (BR). Secretaria de Atenção à Saúde. Departamento de Atenção Básica. Cadernos de Atenção Básica - Saúde sexual e saúde reprodutiva. Brasília (DF): Ministério da Saúde.

Moher, D., Liberati, A., Tetzlaff, J., Altman, D. G., \& The prisma group (2009). Preferred reporting items for systematic reviews and meta-analyses: the PRISMA statement. PLoS Medicine, 6(7), e1000097. 10.1371/journal.pmed.1000097.

Oliveira, B. L. C. A. et al. (2018). Team-Based Learning como Forma de Aprendizagem Colaborativa e Sala de Aula Invertida com Centralidade nos Estudantes no Processo Ensino-Aprendizagem. Rev. bras. educ. med., 42(4), 86-95.

Organização das nações unidas. (1994). Relatório da Conferência Internacional sobre população e Desenvolvimento. Plataforma de Cairo. Cairo: Fundo de População das Nações Unidas (UNFPA-Brasil).

Paiva, C. C. N., \& Caetano, R. (2020) Avaliação de implantação das ações de saúde sexual e reprodutiva na Atenção Primária: revisão de escopo. Esc. Anna Nery, 24(1), e20190142.

Parmejiani, E. P., Queiroz, A. B. A., Pinheiro, A. S., Cordeiro, E. M., Moura, M. A. V., \& Paula, M. B. M. (2021) Saúde sexual e saúde reprodutiva da população ribeirinha: revisão integrativa. Rev Esc Enferm USP. 2021, 55:e03664. https://doi.org/10.1590/S1980-220X2019033103664

Ramos, L. M. H., \& Araujo, R. F. R. (2017) Uso de cartilha educacional sobre diabetes mellitus no processo de ensino e aprendizagem, Ensino, Saúde e Ambiente, 10(3), 94-105.

Rocha, A. C. F. et al. (2016) Atividades de enfermagem no planejamento familiar: projeto de extensão universitária. Extensão em Ação, 1(13).

Santos, J. M. J. et al. (2019). Influência do planejamento reprodutivo e da satisfação materna com a descoberta da gravidez na qualidade da assistência prénatal no Brasil. Rev. Bras. Saúde Mater. Infant., 19(3), 529-535

Santos, R. B. et al. (2016) Processo de readequação de um planejamento familiar: construção de autonomia feminina em uma unidade básica de saúde no Ceará. RECIIS, 10(3), 1-10, 2016.

Souza, M. T., \& Silva, M. D., Carvalho, R. (2010). Revisão integrativa: o que é e como fazer. Einstein. 8(1 Pt 1):102-6.

Telo, S. H. V., \& Witt, R. R. (2018). Saúde sexual e reprodutiva: competências da equipe na Atenção Primária à Saúde. Ciência \& Saúde Coletiva, 23(11), 3481-3490.

WHO. World Health Organization. (2015) Sexual health, human rights and the law. Human reproduction programme. 